\title{
Moduli spaces of germs of holomorphic foliations in the plane
}

David Marín

\begin{abstract}
In this paper we study the topological moduli space of some germs of singular holomorphic foliations in $\left(\mathbb{C}^{2}, 0\right)$. We obtain a fully characterization for generic foliations whose vanishing order at the origin is two or three. We give a similar description for a certain subspace in the moduli space of generic germs of homogeneous foliations of any vanishing order and also for generic quasi-homogeneous foliations. In all the cases we identify the fundamental group of these spaces using the Gassner representation of the pure braid group and a suitable holonomy representation of the foliation.
\end{abstract}

Mathematics Subject Classification (2000). 57R30, 32G.

Keywords. Moduli space, holomorphic foliation, holonomy representation.

\section{Introduction}

Roughly speaking, the moduli space of a germ of a singular holomorphic foliation $\mathcal{F}$ in $\left(\mathbb{C}^{2}, 0\right)$ is the set of holomorphic foliations which are topologically the same as $\mathcal{F}$, but identifying two such foliations that became the same after a holomorphic change of coordinates. Later we will give a precise definition of this object which is quite natural in the classification theory of singularities for holomorphic foliations. However, since it is very difficult to work with, there is an easier but less general way to treat this problem: the use of topologically trivial deformations, i.e. families of topologically equivalent foliations depending continuously of a real parameter $t$. This is the approach of D. Cerveau and P. Sad in [4] where they treat a weak moduli problem: to determine the space of analytic classes of those foliations which can be immersed into a topologically trivial deformation of $\mathcal{F}$. Due to the parameter $t$ in the deformation they called to this object the $t$-moduli space of $\mathcal{F}$, and most of its results treat about it. This seminal work [4] has clearly motivated the present paper. Our purpose is to treat the original moduli problem, i.e. without using deformations, and to prove similar results to the ones that they obtain in the weak problem.

In section 1 we recall some definitions and notations that we will use in the sequel, including the main tool: the projective holonomy representation. Also we 
state the main results that we have obtained. In section 2 we recall the analytic classification theorem for homogeneous foliations. The first original result is obtained in section 3 . There it is shown that two topologically conjugated general foliations have topologically conjugated holonomy representations. In section 4 we prove a topological classification theorem for generic homogenous foliations. In section 5 we identify its moduli space and we endow it with a natural topology. We prove that its fundamental group can be interpreted in terms of certain symmetries of the holonomy representation. Also we show that this group is isomorphic to certain subgroup of the pure braid group of the plane. In section 6 we relate the faithfulness of the Gassner representation of the braid group to the fact that generically the moduli space is simply connected. Finally, in section 7 we generalize the exposed techniques and results to the class of quasi-homogeneous foliations.

I would like to thank my advisors, Marcel Nicolau and Frank Loray, for having guided me so well all over these years, Dominique Cerveau for many discussions and explanations and the referee for several helpful suggestions and comments which made the original manuscript more clear and readable.

\section{Definitions and results}

\section{Definitions}

From now on, $\Phi:\left(\mathbb{C}^{2}, 0\right) \rightarrow\left(\mathbb{C}^{2}, 0\right)$ will denote a germ of homeomorphism conjugating two germs $\mathcal{F}$ and $\mathcal{F}^{\prime}$ of holomorphic foliations at the origin, i.e. $\Phi$ maps leaves of $\mathcal{F}$ onto leaves of $\mathcal{F}^{\prime}$. Moreover we suppose that $\Phi$ preserves the orientation of the ambient space and also preserves leaves orientations. By $\mathcal{F} \sim_{\text {top }} \mathcal{F}^{\prime}$ we mean that there is a conjugating homeomorphism $\Phi$ as above.

Definition 1.1. The moduli space of a germ of holomorphic foliation $\mathcal{F}$ is the following quotient space:

$$
\mathcal{M}(\mathcal{F})=\left\{\mathcal{F}^{\prime} \text { germ of holomorphic foliation } \mid \mathcal{F}^{\prime} \sim_{\text {top }} \mathcal{F}\right\} / \sim_{\text {an }} .
$$

We say that the foliation $\mathcal{F}$ is topologically rigid if $\mathcal{F}^{\prime} \sim_{\text {top }} \mathcal{F}$ implies $\mathcal{F}^{\prime} \sim_{\text {an }} \mathcal{F}$, i.e. $\mathcal{M}(\mathcal{F})=\{[\mathcal{F}]\}$.

Example 1.2. Assume that $\mathcal{F}$ is defined by an holomorphic 1-form with non-zero diagonalizable linear part. We denote by $\alpha$ the ratio of their eigenvalues (called residue of the singularity). If $\alpha$ is non real, the singularity of $\mathcal{F}$ is called hyperbolic. It is well-known that in this case $\mathcal{M}(\mathcal{F})$ can be identified to the upper half plane $\{\alpha \in \mathbb{C} \mid \operatorname{Im}(\alpha)>0\}$.

Seidenberg's desingularisation theorem (see for instance [9]) provides an algorithm of reduction of singularities for every germ of a holomorphic singular folia- 
tion $\mathcal{F}$. We resume it here: after a suitable composition $\pi: \widetilde{\mathbb{C}^{2}} \rightarrow \mathbb{C}^{2}$ of a finite number of blow-ups, all the singularities of $\widetilde{\mathcal{F}}=\pi^{*} \mathcal{F}$ have non-zero linear part and its residue $\alpha$ is not a negative rational number (these singularities are called reduced). In particular, hyperbolic singularities are reduced. We can consider Example 1.2 as the zero step in the study of moduli spaces of holomorphic foliations according to Seidenberg's theorem. The following step is to consider a foliation $\mathcal{F}$ whose reduction of singularities is achieved after a single blow-up $\pi: \widetilde{\mathbb{C}^{2}} \rightarrow \mathbb{C}^{2}$ of the origin. We denote by $D=\pi^{-1}(0)$ the exceptional divisor and assume that $D^{*}=D \backslash \operatorname{Sing}(\widetilde{\mathcal{F}})$ is a leaf of $\widetilde{\mathcal{F}}=\pi^{*} \mathcal{F}$. Let $b$ be a regular point in $D^{*}$ and $\Sigma$ a small analytic section through $b$ transverse to $\widetilde{\mathcal{F}}$. For any loop $\gamma$ in $D^{*}$ based on $b$ there is a germ of a holomorphic return map $H_{\mathcal{F}}(\gamma):(\Sigma, b) \rightarrow(\Sigma, b)$ which only depends on the homotopy class of $\gamma$ in the fundamental group $\Gamma=\pi_{1}\left(D^{*}, b\right)$. The map $H_{\mathcal{F}}: \Gamma \rightarrow \operatorname{Diff}(\Sigma, b)$ is known as the projective holonomy representation of $\mathcal{F}$. If we fixe $\mathcal{F}, b, \Sigma$ and a holomorphic parametrization $(\mathbb{C}, 0) \rightarrow(\Sigma, b)$ then we will write $H: \Gamma \rightarrow \operatorname{Diff}(\mathbb{C}, 0)$ instead to $H_{\mathcal{F}}: \pi_{1}\left(D^{*}, b\right) \rightarrow \operatorname{Diff}(\Sigma, b)$. The image of $H$ in $\operatorname{Diff}(\mathbb{C}, 0)$ is well-defined up to conjugation and it is known as the (projective) holonomy group of $\mathcal{F}$.

We recall the definition of general and N.A.G. foliations given in [4] which are subfamilies of the class of foliations described above.

Definition 1.3. Let $\mathcal{F}$ be a germ of foliation defined by a local 1 -form $\omega=\omega_{\nu}+$ $\omega_{\nu+1}+\cdots$ with $\omega_{\nu} \neq 0$, where $\omega_{k}$ is a homogeneous 1 -form of degree $k \geq \nu=$ vanishing order of $\mathcal{F}$ at 0 . We say that $\omega$ or $\mathcal{F}$ is general if $\omega_{\nu}$ satisfies the following conditions:

(i) If $R=x \frac{\partial}{\partial x}+y \frac{\partial}{\partial y}$ is the radial vector field then the homogeneous polynomial $P_{\nu+1}=\omega_{\nu}(R)$ is non-zero and has $\nu+1$ simple linear factors $l_{1}, \ldots, l_{\nu+1}$.

(ii) The meromorphic 1 -form $\frac{\omega_{\nu}}{P_{\nu+1}}$ has non-real residues $\alpha_{1}, \ldots, \alpha_{\nu+1}$ given by

$$
\frac{\omega_{\nu}}{P_{\nu+1}}=\sum_{j=1}^{\nu+1} \alpha_{j} \frac{d l_{j}}{l_{j}} .
$$

We remark that $\sum_{j=1}^{\nu+1} \alpha_{j}=1$.

We say that $\mathcal{F}$ is N.A.G. (Non Abélien Général) if and only if satisfies the following:

(i) the 1 -form $\omega_{\nu}$ is general,

(ii) the residues $\alpha_{1}, \ldots, \alpha_{\nu+1}$ are different and generate a dense additive subgroup of $\mathbb{C}$,

(iii) the holonomy group of $\mathcal{F}$ is non abelian.

Remark 1.4. N.A.G. conditions imply that the holonomy group $G \subset \operatorname{Diff}(\mathbb{C}, 0)$ is topologically rigid, i.e. if $\psi:(\mathbb{C}, 0) \rightarrow(\mathbb{C}, 0)$ is a germ of homeomorphism such that for any $g \in G$ the composition $\psi \circ g \circ \psi^{-1}$ is an element of $\operatorname{Diff}(\mathbb{C}, 0)$ then $\psi$ is a conformal or anticonformal mapping, cf. [4, 5]. In particular, if $\psi$ preserves the orientation then $\psi$ is a biholomorphism. We note that N.A.G. conditions are 
generic into the space of $(\nu+1)$-jets of 1 -forms $\omega_{\nu}+\omega_{\nu+1}$. Moreover, N.A.G. conditions are invariant under topological conjugations, i.e. if $\mathcal{F}$ is N.A.G. and $\mathcal{F}^{\prime} \sim_{\text {top }} \mathcal{F}$ then $\mathcal{F}^{\prime}$ is also N.A.G., see $[2,4]$.

If $\mathcal{F}$ is a germ of general foliation having vanishing order $\nu$ at the origin then the induced foliation $\widetilde{\mathcal{F}}=\pi^{*} \mathcal{F}$ after blowing-up the origin of $\mathbb{C}^{2}$ has $\nu+1$ different singularities on the exceptional divisor $D$ with hyperbolic linear parts and residues $\alpha_{j}$. Therefore $\mathcal{F}$ is a non dicritical generalized curve (cf. [2]) having $\nu+1$ smooth transverse separatrices through the origin, and it reduces after one blow-up.

Remark 1.5. It is well-known that the germ of four (or less) smooth and transverse curves through the origin is analytically equivalent to the germ of their tangent lines. However, in general this assertion is false for more than four curves.

Definition 1.6. A germ of holomorphic foliation $\mathcal{F}$ which is a non dicritical generalized curve will be called homogeneous if the germ of their separatrices $\operatorname{Sep}(\mathcal{F})$ is analytically equivalent to a finite number of lines through the origin.

In [4] the authors prove the following result.

Theorem (Cerveau-Sad). Let $\mathcal{F}_{0}$ be a general germ of holomorphic foliation in $\left(\mathbb{C}^{2}, 0\right)$. Assume that $\mathcal{F}_{t}$ is a topologically trivial deformation of $\mathcal{F}_{0}$. Then the holonomy representations of $\mathcal{F}_{0}$ and $\mathcal{F}_{t}$ are topologically conjugated.

The assumption on the topologically triviality of the deformation $\mathcal{F}_{t}$ means that there exists a continuous family $\Phi_{t}$ of homeomorphisms of $\left(\mathbb{C}^{2}, 0\right)$ conjugating $\mathcal{F}_{t}$ and $\mathcal{F}_{0}$. In the same paper they conjectured this result without the assumption on the existence of a topologically trivial deformation:

Conjecture (Cerveau-Sad). Let $\mathcal{F}$ and $\mathcal{F}^{\prime}$ be two germs of holomorphic foliations in $\left(\mathbb{C}^{2}, 0\right)$. If $\mathcal{F}$ and $\mathcal{F}^{\prime}$ are topologically conjugated then the holonomy representations of $\mathcal{F}$ and $\mathcal{F}^{\prime}$ are also topologically conjugated.

\section{Main results}

We start with a positive answer to the last conjecture for germs of general holomorphic foliations.

Theorem A. Let $\mathcal{F}$ and $\mathcal{F}^{\prime}$ be two germs of general holomorphic foliations in $\left(\mathbb{C}^{2}, 0\right)$. If $\mathcal{F}$ and $\mathcal{F}^{\prime}$ are topologically conjugated then the holonomy representations of $\mathcal{F}$ and $\mathcal{F}^{\prime}$ are also topologically conjugated.

The next result is a criterion for topological conjugation between germs of 
N.A.G. foliations.

Theorem B. Let $\mathcal{F}$ and $\mathcal{F}^{\prime}$ be two germs of N.A.G. foliations in $\left(\mathbb{C}^{2}, 0\right)$. Then $\mathcal{F}$ is topologically conjugated to $\mathcal{F}^{\prime}$ if and only if there is a geometric isomorphism $\varphi: \Gamma \rightarrow \Gamma^{\prime}$ and a germ of biholomorphism between two transversal sections $\psi$ : $(\Sigma, b) \rightarrow\left(\Sigma^{\prime}, b^{\prime}\right)$ such that $\psi_{*} \circ H_{\mathcal{F}}=H_{\mathcal{F}^{\prime}} \circ \varphi$ and the residues of $\widetilde{\mathcal{F}}$ and $\widetilde{\mathcal{F}}^{\prime}$ at the singularities corresponding by $\varphi$ are equal, where $\psi_{*}$ denotes the conjugation by $\psi$.

Using Theorem B we can study the moduli space of germs of N.A.G. foliations. Next we state the results we have obtained for foliations having vanishing order $\nu=2$ or $\nu=3$ at the origin.

Theorem C. Let $\mathcal{F}$ be a germ of a N.A.G. foliation having vanishing order $\nu=2$ at the origin. Then $\mathcal{F}$ is topologically rigid, i.e. $\mathcal{M}(\mathcal{F})=\{[\mathcal{F}]\}$.

Theorem D. Let $\mathcal{F}$ be a germ of a N.A.G. foliation having vanishing order $\nu=3$ at the origin. Then the moduli space of $\mathcal{F}$ is a connected covering of $\mathbb{C} \backslash\{0,1\}$ whose fundamental group is either trivial or isomorphic to $\mathbb{Z}$, and it is completely determined by the holonomy representation of $\mathcal{F}$. Furthermore, the generic case is the first one, i.e. generically we have $\mathcal{M}(\mathcal{F}) \cong \mathbb{D}$.

A general result (Theorem E) has been obtained in section 5 for homogeneous N.A.G. foliations of any vanishing order $\nu$. However, its formulation needs some definitions that will be introduced later. We have also treated the case of quasihomogeneous foliations in section 7 . There we have applied similar ideas to the homogeneous case, obtaining the Theorem F.

We note that these results are consistent with the ones obtained in [11] for generic germs of foliations (whose singularities can be much more complicated that in our situation). However, its approach is local, i.e. they show the following. Let $\left\{\mathcal{F}_{t}\right\}_{t \in(\mathbb{C}, 0)}$ be a holomorphic deformation of a foliation $\mathcal{F}_{0}$. Suppose that $\mathcal{F}_{t}$ is topologically trivial and $\mathcal{F}_{0}$ verifies some generic conditions. Then there exists a codimension one holomorphic foliation $\mathcal{G}$ in $\left(\mathbb{C}^{3}, 0\right)$ such that $\mathcal{G}_{\mid \mathbb{C}^{2} \times\{t\}}=\mathcal{F}_{t}$. Such a foliation $\mathcal{G}$ is called an (equisingular) unfolding of $\mathcal{F}_{0}$. On the other hand, it is shown that the quotient space of the set of equisingular unfoldings of $\mathcal{F}_{0}$ by the analytic equivalence relation is a neighborhood of the origin in $\mathbb{C}^{\delta\left(\mathcal{F}_{0}\right)}$. For instance, if $\mathcal{F}_{0}$ is a N.A.G. foliation whose vanishing order at the origin is $\nu \geq 2$ then $\delta\left(\mathcal{F}_{0}\right)=\frac{(\nu-1)(\nu-2)}{2}$. This gives us the local description of the moduli space considered in Theorems $\mathrm{C}$ and $\mathrm{D}$.

\section{Analytical classification of homogeneous foliations}

Let $\mathcal{F}$ be a germ of holomorphic foliation at the origin of $\mathbb{C}^{2}$. We will denote by $U$ an open neighborhood of the origin where $\mathcal{F}$ is well-defined. Also we write $U^{*}$ 
to designate the complement in $U$ of the set of separatrices of $\mathcal{F}$. It is well-known that any homogeneous foliation $\mathcal{F}$ can be obtained in the open set $U^{*}$ suspending the holonomy representation $H_{\mathcal{F}}$. Let $\mathcal{F}$ and $\mathcal{F}^{\prime}$ be two given homogeneous foliations whose separatrix sets are analytically equivalent and let $\psi: \Sigma \rightarrow \Sigma^{\prime}$ be a biholomorphic map between two transverse Hopf fibres $\Sigma$ and $\Sigma^{\prime}$, conjugating the holonomies of $\mathcal{F}$ and $\mathcal{F}^{\prime}$. We can apply the lifting path method described in [9] in order to extend $\psi$ to an analytical conjugation $h: U^{*} \rightarrow U^{\prime *}$ between $\mathcal{F}_{\mid U^{*}}$ and $\mathcal{F}_{\mid U^{\prime *}}^{\prime}$, preserving the Hopf fibration. Under a more restrictive hypothesis (the equality of their respective residues) this analytic conjugation $h$ extends to the separatrices of $\mathcal{F}$. Indeed, the following result is proved in [4] using this method.

Theorem (Cerveau-Sad). Let $\mathcal{F}$ and $\mathcal{F}^{\prime}$ be two germs of general homogeneous foliations in $\left(\mathbb{C}^{2}, 0\right)$. Then the following assertions are equivalent:

(i) the foliations $\mathcal{F}$ and $\mathcal{F}^{\prime}$ are analytically conjugated,

(ii) there is a germ of biholomorphism $h:\left(\mathbb{C}^{2}, 0\right) \rightarrow\left(\mathbb{C}^{2}, 0\right)$ preserving the Hopf fibration and conjugating $\mathcal{F}$ and $\mathcal{F}^{\prime}$,

(iii) there exists a biholomorphism $\phi: D \rightarrow D$ such that $\phi(\operatorname{Sing}(\widetilde{\mathcal{F}}))=\operatorname{Sing}\left(\widetilde{\mathcal{F}^{\prime}}\right)$, the residues of $\widetilde{\mathcal{F}}$ and $\widetilde{\mathcal{F}^{\prime}}$ at the singularities corresponding by $\phi$ are equal, and there is a germ of biholomorphism $\psi:(\Sigma, b) \rightarrow\left(\Sigma^{\prime}, b^{\prime}\right)$ verifying the relation $\psi_{*} \circ H_{\mathcal{F}}=H_{\mathcal{F}^{\prime}} \circ \varphi$ with $\varphi=\phi_{*}: \Gamma \rightarrow \Gamma^{\prime}$.

\section{Topological conjugation of holonomies}

The aim of this section is to prove our first result, which we rewrite here more precisely.

Theorem A. Let $\mathcal{F}$ and $\mathcal{F}^{\prime}$ be two germs of general holomorphic foliations in $\left(\mathbb{C}^{2}, 0\right)$. If $\mathcal{F}$ and $\mathcal{F}^{\prime}$ are topologically conjugated then the holonomy representations of $\mathcal{F}$ and $\mathcal{F}^{\prime}$ are topologically conjugated, i.e. there exists a geometric isomorphism $\varphi: \Gamma \rightarrow \Gamma^{\prime}$ and a germ of homeomorphism $\psi:(\Sigma, b) \rightarrow\left(\Sigma^{\prime}, b^{\prime}\right)$ such that $\psi_{*} \circ H_{\mathcal{F}}=$ $H_{\mathcal{F}^{\prime}} \circ \varphi$.

Remark 3.1. Recall that an isomorphism $\varphi: \Gamma \rightarrow \Gamma^{\prime}$ is called geometric if there is an orientation preserving homeomorphism $\phi: D \rightarrow D$ such that $\varphi=\phi_{*}$ is the isomorphism induced by $\phi$ in homotopy.

Let $p: U \backslash\{0\} \rightarrow D$ and $p^{\prime}: U^{\prime} \backslash\{0\} \rightarrow D$ be two $C^{\infty}$ fibrations isotopic to the Hopf fibration such that the separatrices of $\mathcal{F}$ and $\mathcal{F}^{\prime}$ are fibres of $p$ and $p^{\prime}$ respectively. Since these separatrices are smooth holomorphic curves we can assume that $p$ and $p^{\prime}$ are holomorphic fibrations near them. Let $\Phi: U \rightarrow U^{\prime}$ be a homeomorphism conjugating the foliations $\mathcal{F}$ and $\mathcal{F}^{\prime}$ and let $\widetilde{\Phi}: \widetilde{U} \backslash D \rightarrow \widetilde{U^{\prime}} \backslash D$ be the homeomorphism obtained from $\Phi$ after blowing-up the origin in the open 
sets $U$ and $U^{\prime}$. The main difficulty in the proof is the fact that in general there is no homeomorphic extension of $\widetilde{\Phi}$ to the exceptional divisor $D$. We observe that $\widetilde{\Phi}$ preserves the separatrices, so that it sends singularities into singularities along the exceptional divisor (even when it has no extension).

However, in order to clarify the exposition, we first assume that $\widetilde{\Phi}$ can be extended to a homeomorphism $\widetilde{\Phi}: \widetilde{U} \rightarrow \widetilde{U^{\prime}}$ and we denote $\phi=\widetilde{\Phi}_{\mid D}$. Let $\Sigma=$ $p^{-1}(b)$ be a holomorphic fibre of $p$ transverse to $\mathcal{F}$ and close to a separatrix. In general, $\Phi(\Sigma)$ is not a fibre of $p^{\prime}$ but, using a flow-box of $\widetilde{\mathcal{F}^{\prime}}$ centered in $b^{\prime}=\phi(b)$, we can obtain a homeomorphism $\zeta: \Phi(\Sigma) \rightarrow \Sigma^{\prime}=p^{-1}\left(b^{\prime}\right)$. The composition $\psi=\zeta \circ \Phi_{\mid \Sigma}: \Sigma \rightarrow \Sigma^{\prime}$ is a homeomorphism verifying $\psi \circ H_{\mathcal{F}}([\gamma])=H_{\mathcal{F}^{\prime}}([\phi(\gamma)]) \circ \psi$ for any $[\gamma]$ in $\Gamma$. Thus we obtain the desired relation $\psi_{*} \circ H_{\mathcal{F}}=H_{\mathcal{F}^{\prime}} \circ \varphi$ taking as $\varphi=\phi_{*}: \Gamma \rightarrow \Gamma^{\prime}$ the isomorphism induced by the homeomorphism $\phi$.

The argument used in [4] to show the conjugation between the holonomies of $\mathcal{F}$ and $\mathcal{F}^{\prime}$, when the homeomorphism $\widetilde{\Phi}$ does not extend homeomorphically to $D$, needs in an essential way the existence of a topologically trivial deformation $\mathcal{F}_{t}$ joining $\mathcal{F}_{0}=\mathcal{F}$ and $\mathcal{F}_{1}=\mathcal{F}^{\prime}$. Using the compactness of the interval $[0,1]$, it can be assumed that the conjugating homeomorphism $\Phi$ is close to the identity. We recall here the proof given in [4] because the end of the proof in the general case follows the same arguments.

Since $\mathcal{F}$ and $\mathcal{F}^{\prime}$ are close we can choose representatives of a generator system of $\Gamma$ which represents also a generator system in $\Gamma^{\prime}$. Thus, we have a natural isomorphism $\varphi: \Gamma \rightarrow \Gamma^{\prime}$ which is obviously geometric. On the other hand, if $\Phi$ is close enough to the identity then for any compact annulus $C \subset \Sigma \backslash\{b\}$ surrounding the point $b=\Sigma \cap D$ the image of $C$ by $\widetilde{\Phi}$ is contained in an open set of $\widetilde{U^{\prime}}$ whose projection $V^{\prime}$ over $D^{\prime *}$ is simply connected. Thus, $p^{-1}\left(V^{\prime}\right)$ is contained in a flowbox of $\widetilde{\mathcal{F}^{\prime}}$. Therefore, it is easy to construct a homeomorphism $\xi: \widetilde{U^{\prime}} \rightarrow \widetilde{U^{\prime}}$ isotopic to the identity, mapping each leaf of $\widetilde{\mathcal{F}^{\prime}}$ onto itself and such that $\xi(\Phi(C)) \subset \Sigma^{\prime}=$ $p^{\prime-1}\left(b^{\prime}\right)$ for some $b^{\prime}$ in $D^{\prime *}$. We define $\psi_{\mid C}$ as $\xi \circ \Phi_{\mid C}: C \subset \Sigma \rightarrow \Sigma^{\prime}$. Now, we must extend $\psi_{\mid C}$ to the whole $\Sigma$. To do this, we fix a geometric generator system $\gamma_{1}, \ldots, \gamma_{\nu+1}$ of $\Gamma$ such that the holonomy of each generator is contractive (this is possible because all the singularities of $\widetilde{\mathcal{F}}$ are hyperbolic). We choose a compact annulus $C$ in $\Sigma$ containing a fundamental domain $C_{j}$ (and its image $h_{j}\left(C_{j}\right)$ ) of each holonomy generator $h_{j}=H_{\mathcal{F}}\left(\gamma_{j}\right)$. If $z \in \Sigma \backslash C$ there is $n_{j} \in \mathbb{N}$ such that $h_{j}^{-n_{j}}(z)$ belongs to $C_{j}$ and then we can define $\psi_{j}(z)=\left(h_{j}^{\prime}\right)^{n_{j}}\left(\psi\left(h_{j}^{-n_{j}}(z)\right)\right)$, where $h_{j}^{\prime}=H_{\mathcal{F}^{\prime}}\left(\varphi\left(\gamma_{j}\right)\right)$. We will show that $\psi_{i}=\psi_{j}$ gives us a well-defined homeomorphism $\psi: \Sigma \backslash\{b\} \rightarrow \Sigma^{\prime} \backslash\left\{b^{\prime}\right\}$ such that $\psi \circ h_{j}=h_{j}^{\prime} \circ \psi$ in $\Sigma \backslash\{b\}$. Using that the transformations $h_{j}$ and $h_{j}^{\prime}$ are contractive, we can extend continuously $\psi$ to the whole transversal $\Sigma$ mapping $b \in \Sigma \cap D$ into $b^{\prime} \in \Sigma^{\prime} \cap D$. By definition, for any $z \in \Sigma \backslash\{b\}$ we have that

$$
\psi_{i} \circ \psi_{j}^{-1}(z)=h_{i}^{\prime n_{i}} \circ \psi \circ h_{i}^{-n_{i}} \circ h_{j}^{n_{j}} \circ \psi^{-1} \circ h_{j}^{\prime-n_{j}}(z)
$$

with $h_{j}^{\prime-n_{j}}(z) \in C_{j}^{\prime}=\psi\left(C_{j}\right)$ and $h_{i}^{-n_{i}}\left(h_{j}^{n_{j}} \circ \psi^{-1} \circ h_{j}^{\prime-n_{j}}(z)\right) \in C_{i}$. Let us denote 
$x=\psi^{-1} \circ h_{j}^{\prime-n_{j}}(z) \in C_{j}$ and $y=h_{i}^{-n_{i}} \circ h_{j}^{n_{j}}(x) \in C_{i}$. We claim that $\psi(y)=$ $h_{i}^{\prime-n_{i}} \circ h_{j}^{\prime n_{j}}(\psi(x))$ and therefore $\psi_{i} \circ \psi_{j}^{-1}(z)=z$. The claim follows from the facts that $\Phi(C) \subset \Sigma^{\prime}$ and that the foliations $\widetilde{\mathcal{F}}$ and $\widetilde{\mathcal{F}^{\prime}}$ are transversal (outside their separatrices) to the fibrations $p$ and $p^{\prime}$ respectively. In fact, $y$ is the endpoint of a lift $\tilde{\gamma}$ (with respect to the fibration $p$ and beginning at $x$ ) to a leaf of $\widetilde{\mathcal{F}}$ of the loop $\gamma=\gamma_{i}^{-n_{i}} \cdot \gamma_{j}^{n_{j}}$ in $D^{*}$. The path $\tilde{\gamma}^{\prime}=\Phi(\tilde{\gamma})$ has endpoints $\Phi(x)=\psi(x)$ and $\Phi(y)=\psi(y)$ and it is contained in the leaf of $\widetilde{\mathcal{F}^{\prime}}$ passing by $\psi(x)$. By definition of $\varphi$, the projection $p^{\prime}\left(\tilde{\gamma}^{\prime}\right)$ is a loop in $D^{\prime *}$ representing $\varphi([\gamma])=\left[\varphi\left(\gamma_{i}\right)\right]^{-n_{i}} \cdot\left[\varphi\left(\gamma_{j}\right)\right]^{n_{j}}$ in $\Gamma^{\prime}$. Since $\tilde{\gamma}^{\prime}$ is homotopic to the lift of $\varphi([\gamma])$ to the leaf of $\widetilde{\mathcal{F}^{\prime}}$ through $\psi(x)$, we conclude that $\psi(y)=h_{j}^{\prime-n_{j}} \circ h_{i}^{\prime n_{i}}(x)$.

We proceed now to prove Theorem A in the general case. We will show the existence of a geometric isomorphism $\varphi: \Gamma \rightarrow \Gamma^{\prime}$ and a homeomorphism $\xi: \widetilde{U}^{\prime} \rightarrow$ $\widetilde{U^{\prime}}$ such that $p_{*}^{\prime} \circ \Phi_{*}=\varphi \circ p_{*}, \xi$ preserves the foliation $\widetilde{\mathcal{F}}^{\prime}$ and maps a $\widetilde{\Phi}(C)$ into a fibre of $p^{\prime}$, where $C$ is a compact annulus in $\Sigma$ surrounding the point $b$. Then the proof ends as above. Let us consider the following diagram:

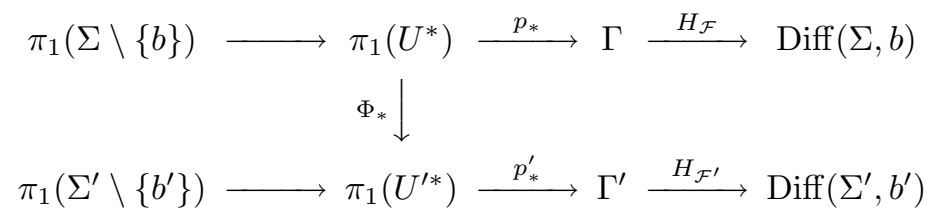

where $\Sigma$ and $\Sigma^{\prime}$ are the fibres of $p$ and $p^{\prime}$ over the points $b \in D^{*}$ and $b^{\prime} \in$ $D^{\prime *}$ (recall that $U^{*}$ and $U^{\prime *}$ denote the complement of separatrices of $\mathcal{F}$ and $\mathcal{F}^{\prime}$ in $U$ and $U^{\prime}$ respectively). The important point is the following: although the homeomorphism $\phi: D \rightarrow D$ does not exist in general the mapping $\widetilde{\Phi}$ induces a well-defined isomorphism $\Gamma \rightarrow \Gamma^{\prime}$. The fundamental group of $U^{*}$ is isomorphic to $\Gamma \oplus \mathbb{Z} c$ where $c$ is a free generator of $\pi_{1}(\Sigma \backslash\{b\})$. Since $\Gamma$ is a free group of rank $\nu \geq 2$, the center of $\pi_{1}\left(U^{*}\right)$ is $\mathbb{Z} c$. We also consider the same situation for $\mathcal{F}^{\prime}$. Since $\Phi_{*}$ is an isomorphism, it maps the center $\mathbb{Z} c$ onto the center $\mathbb{Z} c^{\prime}$ of $\pi_{1}\left(U^{\prime *}\right)$. Therefore $\Phi_{*}$ induces an isomorphism $\varphi: \Gamma \rightarrow \Gamma^{\prime}$ such that $p_{*}^{\prime} \circ \Phi_{*}=\varphi \circ p_{*}$. Now, we fix two systems of geometric generators of $\Gamma$ and $\Gamma^{\prime}$. Let $\gamma$ be a loop in $D^{*}$ based on $b$ of type $\gamma=\delta \cdot \epsilon \cdot \delta^{-1}$ representing a geometric generator in $\Gamma$, where $\epsilon$ is a loop surrounding closely some singularity of $\widetilde{\mathcal{F}}$. Let $\tilde{\gamma}$ be a loop in $\widetilde{U^{*}}$ of the same type than $\gamma$ such that $p(\tilde{\gamma})=\gamma$. Thus, we can write $\varphi([\gamma])=\left[p^{\prime}(\Phi(\tilde{\gamma}))\right]$ also in the form $\left[\delta^{\prime} \cdot \epsilon^{\prime} \cdot \delta^{\prime-1}\right]$. Therefore $\varphi$ maps every geometric generator of $\Gamma$ into a conjugate of a geometric generator of $\Gamma^{\prime}$. By Nielsen's theorem (see for instance the first chapter of [13]) we conclude that $\varphi$ is a geometric isomorphism, i.e. it is induced by a homeomorphism which a priori is defined at the singularities. The precedent considerations reduce the proof of Theorem A to prove the following lemma.

Lemma 3.2. If $\Sigma$ is contained in a small enough neighborhood of a separatrix of $\mathcal{F}$ and $C$ is a compact annulus contained in $\Sigma$ and surrounding $D$, then there exists a homeomorphism $\xi: \widetilde{U^{\prime}} \rightarrow \widetilde{U^{\prime}}$ isotopic to the identity, preserving the foliation $\widetilde{\mathcal{F}}^{\prime}$ 
and such that $\xi(\Phi(C)) \subset \Sigma^{\prime}$.

Proof. Let $S_{0}$ be a separatrix of $\widetilde{\mathcal{F}}$ through a singular point $t_{0} \in D$ and let $V^{\prime}$ be a neighborhood of $S_{0}^{\prime}=\Phi\left(S_{0}\right)$ admitting a local coordinate system $\left(x^{\prime}, y^{\prime}\right)$ which linearizes the singularity $t_{0}^{\prime} \in S_{0}^{\prime} \cap D$. We consider a compact annulus $C_{0}$ contained in $S_{0} \backslash\left\{t_{0}\right\}$ whose outer radius is small enough so that $C_{0}^{\prime}=\Phi\left(C_{0}\right)$ is contained in $V^{\prime}$. Let $W^{\prime} \subset V^{\prime}$ be a tubular neighborhood of $C_{0}^{\prime}$ disjoint of $D$ and let $W$ be a tubular neighborhood of $C_{0}$ such that $\Phi(W) \subset W^{\prime}$. We consider a $p$-fibre $\Sigma$ close enough to $S_{0}$ so that $W \cap \Sigma$ contains a compact annulus $C$ with the desired size. Therefore $\Phi(C) \subset W^{\prime}$. The foliation $\widetilde{\mathcal{F}}_{\mid V^{\prime}}$ is given by a linear vector field $X_{0}^{\prime}=x^{\prime} \frac{\partial}{\partial x^{\prime}}-\alpha_{0} y^{\prime} \frac{\partial}{\partial y^{\prime}}$ and the projection $p_{\mid V^{\prime}}^{\prime}$ by $\left(x^{\prime}, y^{\prime}\right) \mapsto x^{\prime}$. Let $\Sigma^{\prime}=\left\{x^{\prime}=x_{0}^{\prime}\right\}$ be a fibre of $p^{\prime}$ contained in $V^{\prime}$. We consider the map $f=p^{\prime} \circ \Phi_{\mid C}: C \rightarrow D^{\prime *}$ which is continuous and null-homotopic because the homotopy type of $C$ generates the kernel of $p_{*}$. Therefore there exists a continuous logarithm of $f$ in $C$. Since the flow of $X_{0}^{\prime}$ is given by $\varphi_{T}\left(x^{\prime}, y^{\prime}\right)=\left(x^{\prime} e^{T}, y^{\prime} e^{-\alpha_{0} T}\right)$ we can define $\xi_{\mid \Phi(C)}$ as the flow of $X_{0}^{\prime}$ at time $T=\log x_{0}^{\prime}-\log \left(f \circ \Phi^{-1}\right)$. Using a test function it is easy to extend $\xi$ as a global homeomorphism of $\widetilde{U^{\prime}}$ such that coincides with the identity map out of $W^{\prime}$ and verifies the conditions of the lemma.

\section{Topological classification of N.A.G. foliations}

We proceed to prove Theorem B. We recall (see Remark 1.4) that N.A.G. conditions imply the rigidity of the holonomy group of $\mathcal{F}$. Therefore, under these hypothesis, the homeomorphism $\psi: \Sigma \rightarrow \Sigma^{\prime}$ constructed in Theorem A is actually a biholomorphism. To see the necessity of the conditions expressed in Theorem B for the topological conjugation, we must only prove the following point: The residues of the singularities of $\widetilde{\mathcal{F}}$ and $\widetilde{\mathcal{F}}^{\prime}$ related by $\varphi$ are equal.

For each $j=1, \ldots, \nu+1$, we choose coordinates $y_{j}$ in $\Sigma$ and $y_{j}^{\prime}$ in $\Sigma^{\prime}$ which linearize the holonomy transformations $h_{j}=H_{\mathcal{F}}\left(\gamma_{j}\right)$ and $h_{j}^{\prime}=H_{\mathcal{F}^{\prime}}\left(\varphi\left(\gamma_{j}\right)\right)$ respectively. Then the following relations hold:

$$
\psi\left(e^{-2 i \pi \alpha_{j}} y_{j}\right)=e^{-2 i \pi \alpha_{j}^{\prime}} \psi\left(y_{j}\right) .
$$

Therefore, from (1) we get after differentiation $\psi^{\prime}(0) e^{-2 i \pi \alpha_{j}}=e^{-2 i \pi \alpha_{j}^{\prime}} \psi^{\prime}(0)$, so that we have at once $\alpha_{j}=\alpha_{j}^{\prime}+k_{j}$, for some integer $k_{j}$.

Lemma 4.1. With the notations introduced above we have that all the integers $k_{j}$ are zero.

Proof. For the sake of simplicity we will omit the subindex $j$. Let $\gamma=\left(\gamma_{x}, \gamma_{y}\right)$ be a path contained in a leaf $L$ of $\widetilde{\mathcal{F}}$ which is not a separatrix and let $\gamma^{\prime}=\left(\gamma_{x}^{\prime}, \gamma_{y}^{\prime}\right)$ be a path contained in $\Phi(L)$. Assume that $\gamma_{x}$ and $\gamma_{x}^{\prime}$ are loops with base points 
$b$ and $b^{\prime}$ such that $\varphi\left(\left[\gamma_{x}\right]\right)=\left[\gamma_{x}^{\prime}\right]$. We consider the homotopy class of the loop $\Phi\left(\gamma \cdot \gamma_{y}^{-1}\right)=\Phi(\gamma) \cdot \psi\left(\gamma_{y}\right)^{-1}$ in $\pi_{1}\left(U^{* *}\right)=\Gamma^{\prime} \oplus \mathbb{Z} c^{\prime}$. In fact, this loop belongs to $\Gamma^{\prime} \subset \pi_{1}\left(U^{\prime *}\right)$. Then the $y^{\prime}$-projection of $\Phi(\gamma)$ is homotopic with fixed endpoints to $\psi\left(\gamma_{y}\right)$ in $\Sigma^{\prime *}$. On the other hand, $\Phi(\gamma)$ is homotopic to $\gamma^{\prime}$ because both paths are lifts of homotopic loops in $D^{\prime *}$ to the same leaf $\Phi(L)$. Therefore, the $y^{\prime}$-projection of $\Phi(\gamma)$ is also homotopic to $\gamma_{y}^{\prime}$. We conclude that $\psi\left(\gamma_{y}\right)$ and $\gamma_{y}^{\prime}$ are homotopic with fixed endpoints in $\Sigma^{\prime *}$. It is easy to see that this last assertion implies that $k=0$.

We now proceed to see that the conditions of Theorem B are sufficient to assure that $\mathcal{F}$ and $\mathcal{F}^{\prime}$ are topologically conjugated. Indeed, we will construct a conjugating fibred homeomorphism. Let $D_{i}, D_{i}^{\prime} \subset D$ be small disks around the singularities of $\widetilde{\mathcal{F}}$ and $\widetilde{\mathcal{F}}{ }^{\prime}$ such that $p$ and $p^{\prime}$ are holomorphic over them. Since $\varphi: \Gamma \rightarrow \Gamma^{\prime}$ is a geometric isomorphism there is an orientation preserving homeomorphism $\phi:(D, \operatorname{Sing}(\widetilde{\mathcal{F}})) \rightarrow\left(D, \operatorname{Sing}\left(\widetilde{\mathcal{F}^{\prime}}\right)\right)$ with $\varphi=\phi_{*}$. We can suppose that $\phi_{\mid D_{i}}: D_{i} \rightarrow D_{i}^{\prime}$ is holomorphic for any $i=1, \ldots, \nu+1$. Consider the open sets $\widetilde{V}=\widetilde{U^{*}} \cup D^{*}$ and $\widetilde{V^{\prime}}=\widetilde{U^{\prime *}} \cup D^{\prime *}$. We define $\widetilde{\Phi}: \widetilde{V} \rightarrow \widetilde{V^{\prime}}$ using the lifting path method. More precisely, for any $x \in \widetilde{V}$ we consider a path $\tilde{\gamma}$ contained in the leaf of $\widetilde{\mathcal{F}}$ through $x$ and joining $x$ with a point $y \in \Sigma$. Then $\gamma=p(\tilde{\gamma})$ is a path in $D^{*}$ joining $p(x)$ and $b \in \Sigma \cap D$. Thus $\phi(\gamma)^{-1}$ is a path in $D^{\prime *}$ joining $b^{\prime} \in \Sigma^{\prime} \cap D$ and $\phi(p(x))$ that we can lift to the leaf of $\widetilde{\mathcal{F}^{\prime}}$ through $\psi(y) \in \Sigma^{\prime}$. The endpoint of this lifting is a point $x^{\prime} \in p^{-1}(\phi(p(x)))$ and we put $\widetilde{\Phi}(x)=x^{\prime}$. This definition of $\widetilde{\Phi}(x)$ does not depend on the choice of the path $\tilde{\gamma}$ because the pair $(\psi, \varphi)$ conjugates the holonomy representations of $\mathcal{F}$ and $\mathcal{F}^{\prime}$. On the other hand, since $p$ and $p^{\prime}$ are holomorphic in a neighborhood of the separatrices of $\widetilde{\mathcal{F}}$ and $\mathcal{F}^{\prime}$, as well as $\phi_{\mid D_{i}}: D_{i} \rightarrow D_{i}^{\prime}$ and $\psi: \Sigma \rightarrow \Sigma^{\prime}$, we have that $\widetilde{\Phi}_{\mid p^{-1}\left(D_{i}^{*}\right)}$ is also holomorphic. Using the equality of the residues of $\widetilde{\mathcal{F}}$ and $\widetilde{\mathcal{F}}^{\prime}$, we deduce as in [9] that $\widetilde{\Phi}$ has a holomorphic extension to the separatrices. Thus, we have constructed an orientation preserving homeomorphism $\widetilde{\Phi}: \widetilde{U} \rightarrow \widetilde{U^{\prime}}$ such that

(i) $\widetilde{\Phi}$ is a topological conjugation between $\widetilde{\mathcal{F}}$ and $\widetilde{\mathcal{F}}^{\prime}$;

(ii) $\underset{\Phi}{\widetilde{\Phi}}$ is fibred, i.e. $p \circ \widetilde{\Phi}=\widetilde{\Phi} \circ p^{\prime}$

(iii) $\widetilde{\Phi}_{\mid D}=\phi$ where $\phi_{*}=\varphi: \Gamma \rightarrow \Gamma^{\prime}$ is the given geometric isomorphism;

(iv) $\widetilde{\Phi}_{\mid \Sigma}=\psi: \Sigma \rightarrow \Sigma^{\prime}$ is the given holonomy conjugation.

\section{Moduli space of homogeneous foliations}

In this section we will identify certain subspaces inside the moduli space of a homogeneous foliation, which we precise in the following definition.

Definition 5.1. Let $\mathcal{F}_{0}$ be a homogeneous foliation. Then

$$
\mathcal{M}_{\mathcal{H}}\left(\mathcal{F}_{0}\right):=\left\{\mathcal{F} \text { homogeneous germ of foliation } \mid \mathcal{F} \sim_{\text {top }} \mathcal{F}_{0}\right\} / \sim_{\text {an }} \hookrightarrow \mathcal{M}\left(\mathcal{F}_{0}\right)
$$


is called the moduli space of $\mathcal{F}_{0}$ relative to the homogeneous class.

Remark 5.2. If the vanishing order of $\mathcal{F}_{0}$ is $\nu \leq 3$ then $\mathcal{M}_{\mathcal{H}}\left(\mathcal{F}_{0}\right)=\mathcal{M}\left(\mathcal{F}_{0}\right)$. This follows immediately from Remark 1.5.

By Definition 1.3, the residues of $\mathcal{F}_{0}$ are all different. We enumerate the singularities of $\widetilde{\mathcal{F}}_{0}$ once and for all. If $\mathcal{F}$ is a germ of a holomorphic foliation topologically conjugated to $\mathcal{F}_{0}$ then the residues of $\mathcal{F}$ and $\mathcal{F}_{0}$ are equal. Labelling each singularity of $\widetilde{\mathcal{F}}$ by means of its residue, we induce a well-defined map

$$
\hat{\sigma}:\left\{\mathcal{F} \text { homogeneous } \mid \mathcal{F} \sim_{\text {top }} \mathcal{F}_{0}\right\} \rightarrow F_{\nu+1}\left(\mathbb{S}^{2}\right) .
$$

Here, $F_{\nu+1}\left(\mathbb{S}^{2}\right)$ denotes the set $\left\{\left(t_{1}, \ldots, t_{\nu+1}\right) \in\left(\mathbb{S}^{2}\right)^{\nu+1} \mid t_{i} \neq t_{j}\right.$ if $\left.i \neq j\right\}$ (we use the notation of [1] for the configuration spaces for braid groups). Note that $\operatorname{PSL}(2, \mathbb{C})$ acts on $F_{\nu+1}\left(\mathbb{S}^{2}\right)$ in a natural way, with quotient space

$$
F_{3, \nu-2}\left(\mathbb{S}^{2}\right):=\left\{\left(t_{1}, \ldots, t_{\nu-2}\right) \in\left(\mathbb{S}^{2} \backslash\{0,1, \infty\}\right)^{\nu-2} \mid t_{i} \neq t_{j} \text { if } i \neq j\right\} .
$$

Let $\sigma: \mathcal{M}_{\mathcal{H}}\left(\mathcal{F}_{0}\right) \rightarrow F_{3, \nu-2}\left(\mathbb{S}^{2}\right)$ be the map induced by $\hat{\sigma}$ on the quotient. The following Theorem E identifies the moduli space, relative to the homogeneous class, of a homogeneous N.A.G. foliation with vanishing order $\nu$ at the origin. Theorem $\mathrm{C}$ follows from Theorem E particularizing $\nu=2$ and considering Remark 5.2. Putting $\nu=3$ in Theorem E also we obtain Theorem $\mathrm{D}$, except for the claim on the generic triviality of the fundamental group of $\mathcal{M}\left(\mathcal{F}_{0}\right)$. This last property will be proved in the next section.

Theorem E. Let $\mathcal{F}_{0}$ be a germ of a homogeneous N.A.G. foliation in $\left(\mathbb{C}^{2}, 0\right)$ with vanishing order $\nu \geq 2$. Then $\mathcal{M}_{\mathcal{H}}\left(\mathcal{F}_{0}\right)$ is a connected covering of $F_{3, \nu-2}\left(\mathbb{S}^{2}\right)$ with covering map $\sigma$, and its fundamental group is completely determined by the holonomy representation of $\mathcal{F}_{0}$.

The rest of the section is devoted to proving Theorem E. The idea of the proof is to construct a map $\lambda$ from the universal covering $\tilde{X}$ of $X=F_{3, \nu-2}\left(\mathbb{S}^{2}\right)$ to $\mathcal{M}_{\mathcal{H}}\left(\mathcal{F}_{0}\right)$. This map $\lambda$ will induce a homeomorphism from the quotient space $X / G$ onto $\mathcal{M}_{\mathcal{H}}\left(\mathcal{F}_{0}\right)$ for a certain subgroup $G$ of the fundamental group of $X$, which depends on the holonomy representation of $\mathcal{F}_{0}$.

\subsection{Constructing homogeneous foliations with prescribed holonomy}

We consider the following geometric data:

- $t_{1}, \ldots, t_{\nu+1}$ and $b$ different points in $\mathbb{S}^{2}$,

- $h_{1}, \ldots, h_{\nu+1}$ linearizable germs of holomorphic diffeomorphims such that

$$
h_{1} \circ \cdots \circ h_{\nu+1}=\mathrm{id},
$$


- $\alpha_{1}, \ldots, \alpha_{\nu+1}$ complex numbers such that $\alpha_{1}+\cdots+\alpha_{\nu+1}=1$ and $e^{-2 i \pi \alpha_{j}}=$ $h_{j}^{\prime}(0)$ for $j=1, \ldots, \nu+1$,

- $\delta_{1}, \ldots, \delta_{\nu+1}:[0,1] \rightarrow \mathbb{S}^{2}$ disjoints curves joining $b$ and $t_{1}, \ldots, t_{\nu+1}$ respectively,

We can choose a positive number $\varepsilon>0$ such that if $\hat{\delta}_{j}=\delta_{j \mid[0,1-\varepsilon]}$ and $\hat{\gamma}_{j}$ is a closed curve based on $\delta_{j}(1-\varepsilon)$ then we obtain a generator system $\left\{\gamma_{j}=\left[\hat{\delta}_{j} \hat{\gamma}_{j} \hat{\delta}_{j}^{-1}\right]\right\}_{j=1}^{\nu+1}$ of the fundamental group $\Gamma=\pi_{1}\left(\mathbb{S}^{2} \backslash\left\{t_{1}, \ldots, t_{\nu+1}\right\}, b\right)$ such that $\gamma_{1} \cdots \gamma_{\nu+1}=1$. A theorem of A. Lins Neto in [6] claims that, given the geometric data as above, there exists a germ of homogeneous foliation $\mathcal{F}$ in $\left(\mathbb{C}^{2}, 0\right)$ with separatrices and holonomy representation given by

$$
\operatorname{Sep}(\mathcal{F})=\bigcup_{j=1}^{\nu+1}\left\{y-t_{j} x=0\right\}, \quad H_{\mathcal{F}}: \Gamma \longrightarrow \operatorname{Diff}(\mathbb{C}, 0) \quad \text { with } \quad \gamma_{j} \longmapsto h_{j}
$$

and residue $\alpha_{j}$ at the singularity $t_{j}$. We remark that the analytic class of $\mathcal{F}$ is independent of the choice of the curves $\delta_{j}$ in a fixed homotopy class (with fixed endpoints). From now on, we fix the holonomy data for the foliation $\mathcal{F}_{0}$ : $t_{1}^{0}, \ldots, t_{\nu-2}^{0}, t_{\nu-1}^{0}=0, t_{\nu}^{0}=1, t_{\nu+1}^{0}=\infty ; h_{1}, \ldots, h_{\nu+1} ; \alpha_{1}, \ldots, \alpha_{\nu+1}$ and $\delta_{1}^{0}, \ldots, \delta_{\nu+1}^{0}$.

\subsection{Some topological facts about configuration spaces}

We recall that the map $F_{3, \nu-1}\left(\mathbb{S}^{2}\right) \rightarrow F_{3, \nu-2}\left(\mathbb{S}^{2}\right)$ given by forgetting the base point $b$ of $\left(b, t_{1}, \ldots, t_{\nu-2}\right)$ in $F_{3, \nu-1}\left(\mathbb{S}^{2}\right)$ is a locally trivial bundle whose fibre is $\mathbb{S}^{2} \backslash\left\{t_{1}^{0}, \ldots, t_{\nu+1}^{0}\right\}$. The associated long exact sequence in homotopy finishes in

$$
1 \rightarrow \Gamma \rightarrow \pi_{1}\left(F_{3, \nu-1}\left(\mathbb{S}^{2}\right)\right) \rightarrow \pi_{1}\left(F_{3, \nu-2}\left(\mathbb{S}^{2}\right)\right) \rightarrow 1
$$

Since the bundle $F_{3, \nu-1}\left(\mathbb{S}^{2}\right) \rightarrow F_{3, \nu-2}\left(\mathbb{S}^{2}\right)$ admits a continuous cross-section, the short exact sequence $(2)$ splits. Therefore, the group $\pi_{1}\left(F_{3, \nu-1}\left(\mathbb{S}^{2}\right)\right)$ is the semidirect product of $\Gamma$ and $M_{\nu}=\pi_{1}\left(F_{3, \nu-2}\left(\mathbb{S}^{2}\right)\right)$ by means of a morphism $\varphi: M_{\nu} \rightarrow$ $\operatorname{Aut}(\Gamma)$. We will denote the image by $\varphi$ of $\tau \in M_{\nu}$ by $\varphi_{\tau}$.

Remark 5.3. The group $M_{\nu}$ can be identified with the mapping class group of the sphere $\mathbb{S}^{2}$ minus $\nu+1$ colored points. On the other hand, the morphism $\varphi$ is the restriction of the well-known faithful representation of the pure braid group of the plane $P_{\nu}=\pi_{1}\left(F_{\nu}\left(\mathbb{R}^{2}\right)\right)$ into the automorphism group of the free group of rank $\nu$. Indeed $F_{\nu}\left(\mathbb{R}^{2}\right) \cong F_{2, \nu-2}\left(\mathbb{R}^{2}\right) \times \operatorname{Aff}(1, \mathbb{C})$ and then $P_{\nu} \cong M_{\nu} \oplus \mathbb{Z}$.

Each automorphism in the image of $\varphi$ is induced by a homeomorphism of the sphere onto itself. We will describe it. If $\tau \in M_{\nu}$ is represented by a loop $\left(b^{s}, t_{1}^{s}, \ldots, t_{\nu-2}^{s}\right)$ in $F_{3, \nu-1}\left(\mathbb{S}^{2}\right)$ then there is an isotopy $h_{s}: \mathbb{S}^{2} \rightarrow \mathbb{S}^{2}, h_{0}=i d$ such that $h_{s}\left(b^{0}\right)=b^{s}$ and $h_{s}\left(t_{j}^{0}\right)=t_{j}^{s}$. We put $\varphi_{\tau}=\left(h_{1}\right)_{*}: \Gamma \rightarrow \Gamma$. 


\subsection{Constructing the map $\lambda$}

Let $\tilde{X}$ be the universal covering space of $X=F_{3, \nu-2}\left(\mathbb{S}^{2}\right)$ viewed as homotopy classes of paths with fixed endpoints, starting in $\left(b^{0}, t_{1}^{0}, \ldots, t_{\nu-2}^{0}\right)$ and let $\tau$ be an element of $\widetilde{X}$ represented by a path $\left(b^{s}, t_{1}^{s}, \ldots, t_{\nu-2}^{s}\right)$. As before, there is an isotopy $h_{s}: \mathbb{S}^{2} \rightarrow \mathbb{S}^{2}, h_{0}=i d$ such that $h_{s}\left(b^{0}\right)=b^{s}$ and $h_{s}\left(t_{j}^{0}\right)=t_{j}^{s}$. The homeomorphism

$$
h_{1}:\left(\mathbb{S}^{2} \backslash\left\{t_{1}^{0}, \ldots, t_{\nu+1}^{0}\right\}, b^{0}\right) \rightarrow\left(\mathbb{S}^{2} \backslash\left\{t_{1}^{1}, \ldots, t_{\nu+1}^{1}\right\}, b^{1}\right)
$$

allows us to define new curves $\delta_{j}^{1}=h_{1}\left(\delta_{j}^{0}\right)$ from the old ones. From Lins Neto's Theorem, there is a homogeneous foliation $\mathcal{F}_{\tau}$ associated to this new data and the precedent holonomy transformations and residues. Thanks to Theorem B we conclude that $\mathcal{F}_{\tau}$ is topologically conjugated to $\mathcal{F}_{0}$. Thus we have constructed a well-defined map

$$
\lambda: \widetilde{X} \rightarrow \mathcal{M}_{\mathcal{H}}\left(\mathcal{F}_{0}\right), \quad \tau \mapsto\left[\mathcal{F}_{\tau}\right]
$$

Furthermore, if we write $H_{0}$ for the holonomy representation of $\mathcal{F}_{0}$ then $H_{\tau}=$ $H_{0} \circ\left(h_{1}\right)_{*}^{-1}=H_{0} \circ \varphi_{\tau}^{-1}$ is the holonomy representation of $\mathcal{F}_{\tau}$. By the analytical classification theorem we deduce that $\mathcal{F}_{\tau} \sim_{\text {an }} \mathcal{F}_{\tau^{\prime}}$ if and only if $\sigma(\tau)=\sigma\left(\tau^{\prime}\right)$ and there is a $\psi \in \operatorname{Diff}(\mathbb{C}, 0)$ such that $\psi_{*} \circ H_{\tau}=H_{\tau^{\prime}} \circ(i d)_{*}$ or equivalently $\psi_{*} \circ H_{0}=H_{0} \circ \varphi_{\tau \tau^{\prime-1}}$. We consider the following subgroup of $M_{\nu}$ :

Definition 5.4. We define the symmetry group of the representation $H_{0}$ by $\operatorname{Sim}\left(H_{0}\right)=\left\{\tau \in M_{\nu} \mid\right.$ there exists $\psi \in \operatorname{Diff}(\mathbb{C}, 0)$ such that $\left.\psi_{*} \circ H_{0}=H_{0} \circ \varphi_{\tau}\right\}$.

From the precedent considerations follows that the map $\lambda$ induces an injective $\operatorname{map} \bar{\lambda}: \widetilde{X} / \operatorname{Sim}\left(H_{0}\right) \rightarrow \mathcal{M}_{\mathcal{H}}\left(\mathcal{F}_{0}\right)$ which is also surjective by Theorem B. Moreover $\sigma=\bar{\lambda}^{-1} \circ \pi$. In order to prove that $\sigma$ is a covering map it suffices to show that $\bar{\lambda}$ is a homeomorphism. We will endow $\mathcal{M}_{\mathcal{H}}\left(\mathcal{F}_{0}\right)$ (actually $\mathcal{M}\left(\mathcal{F}_{0}\right)$ ) with a natural topology which will make $\lambda$ a continuous open map.

According to Theorem B, if $\mathcal{F}$ and $\mathcal{F}^{\prime}$ are topologically conjugated then there is a fibred homeomorphism $\Phi$ which induces a homeomorphism $\phi$ on the exceptional divisor $D \cong \mathbb{S}^{2}$. We endow the group of homeomorphisms of $\mathbb{S}^{2}$ with the compactopen topology. We fix a holomorphic foliation $\mathcal{F}$ topologically conjugated to $\mathcal{F}_{0}$. For any neighborhood $V$ of the identity map on $\mathbb{S}^{2}$ and for any neighborhood $U$ of $\sigma([\mathcal{F}])$ in $X=F_{3, \nu-2}\left(\mathbb{S}^{2}\right)$ we put

$$
\begin{aligned}
W_{\mathcal{F}}(U, V)=\left\{\left[\mathcal{F}^{\prime}\right] \in\right. & \sigma^{-1}(U) \text { such that } \mathcal{F} \text { and } \mathcal{F}^{\prime} \text { are conjugated by a } \\
& \text { fibred homeomorphism inducing } \phi \in V \text { on } D\} .
\end{aligned}
$$

It can be easily verified that there is a topology for which $\left\{W_{\mathcal{F}}(U, V)\right\}_{U, V}$ is a base of neighborhoods of $[\mathcal{F}]$ in $\mathcal{M}\left(\mathcal{F}_{0}\right)$. This topology makes $\lambda: \widetilde{X} \rightarrow \mathcal{M}_{\mathcal{H}}\left(\mathcal{F}_{0}\right)$ both continuous and open.

Remark 5.5. The transverse action of the conjugating homeomorphism $\Phi$ between $\mathcal{F}$ and $\mathcal{F}^{\prime}$ is not considered in the definition of this topology because $\Phi$ is 
transversally holomorphic by N.A.G. conditions imposed on $\mathcal{F}_{0}$.

Thus we have shown that $\mathcal{M}_{\mathcal{H}}\left(\mathcal{F}_{0}\right)$ is homeomorphic to $\widetilde{X} / \operatorname{Sim}\left(H_{0}\right)$. Consequently, the fundamental group of $\mathcal{M}_{\mathcal{H}}\left(\mathcal{F}_{0}\right)$ is isomorphic to $\operatorname{Sim}\left(H_{0}\right)$.

Remark 5.6. There is another approach to study the moduli space of a germ of a holomorphic foliation $\mathcal{F}_{0}$ in the spirit of the Teichmüller theory for Riemann surfaces. Using this approach the universal covering of $F_{3, \nu-2}\left(\mathbb{S}^{2}\right)$ can be viewed as the Teichmüller space of $\mathcal{F}_{0}$ and $\operatorname{Sim}\left(H_{0}\right)$ becomes the modular group of $\mathcal{F}_{0}$.

\section{Symmetry group of the holonomy representation}

Let $\mathcal{F}_{0}$ be a germ of a N.A.G. holomorphic foliation with holonomy representation $H_{0}: \Gamma \rightarrow \operatorname{Diff}(\Sigma, b)$ and let $\left(\gamma_{1}, \ldots, \gamma_{\nu+1}\right)$ be a geometric generator system of $\Gamma$ as in the section 5.1. We can choose a transverse coordinate $z$ on $\Sigma$ which linearizes the holonomy transformation $h_{\nu+1}=H_{0}\left(\gamma_{\nu+1}\right)$. We want to study the properties of the symmetry group of $H_{0}$ defined in 5.4. Indeed, we can define the symmetry group of any representation $\rho$ of $\Gamma$ in an abstract group $G$ in a natural way:

$$
\operatorname{Sim}(\rho)=\left\{\tau \in M_{\nu} \mid \text { there exists } g \in G \text { such that } I_{g} \circ \rho=\rho \circ \varphi_{\tau}\right\},
$$

where $I_{g}: G \rightarrow G$ denotes the inner automorphism of $G$ associated to $g$. Note that if $\rho: \Gamma \rightarrow G$ and $\rho^{\prime}: \Gamma \rightarrow G^{\prime}$ are related by $\rho^{\prime}=f \circ \rho$ where $f: G \rightarrow G^{\prime}$ is an isomorphism then $\operatorname{Sim}(\rho)=\operatorname{Sim}\left(\rho^{\prime}\right)$. In this case we will say that $\rho$ and $\rho^{\prime}$ are conjugated by $f$.

For any integer $k \geq 1$ we apply this definition to the $k$-jet transformation group $G_{k}=J^{k} \operatorname{Diff}(\mathbb{C}, 0)$ and the $k$-truncated representation $\rho_{k}=J^{k} H_{0}$ of $\Gamma$. We obtain a chain of inclusions

$$
M_{\nu} \supseteq \operatorname{Sim}\left(\rho_{1}\right) \supseteq \operatorname{Sim}\left(\rho_{2}\right) \supseteq \cdots \supseteq \operatorname{Sim}\left(\rho_{k}\right) \supseteq \operatorname{Sim}\left(\rho_{k+1}\right) \supseteq \cdots
$$

verifying the equality $\operatorname{Sim}\left(H_{0}\right)=\bigcap_{k \geq 1} \operatorname{Sim}\left(\rho_{k}\right)$. Since $\mathcal{F}_{0}$ is a N.A.G. foliation, its holonomy group is not abelian and then there is a first integer $k_{0} \geq 2$ such that the image $\rho_{k_{0}}(\Gamma)$ is a non abelian group, i.e. in such a way that there is an element $\gamma \in \Gamma$ verifying $H_{0}(\gamma): z \mapsto \alpha z+\beta z^{k_{0}}+\cdots$ with $\beta \neq 0$. We remark that, by definition, the integer $k_{0}$ only depends on the representation $H_{0}$ and the choice of the linearizing coordinate $z$. We check easily that $\rho_{k_{0}}$ is conjugated to an affine representation $\rho: \Gamma \rightarrow \operatorname{Aff}(1, \mathbb{C})=\left\{\zeta \mapsto a \zeta+b, a \in \mathbb{C}^{*}, b \in \mathbb{C}\right\}$ by the isomorphism

$$
j^{k_{0}}\left(\alpha z+\beta z^{k_{0}}+\cdots\right) \longmapsto\left(\zeta \mapsto \alpha^{1-k_{0}} \zeta+\alpha^{-k_{0}} \beta\right),
$$

which is induced by the coordinate change $z=\left(1-k_{0}\right) \zeta^{1-k_{0}}$. In addition, the choice of the coordinate $z$ (linearizing $h_{\nu+1}$ ) implies that if $\rho\left(\gamma_{i}\right)=a_{i} \zeta+b_{i}$ for $i=1, \ldots, \nu+1$ then $b_{\nu+1}=0$ and the translation vector $\vec{b}=\left(b_{1}, \ldots, b_{\nu}\right) \in \mathbb{C}^{\nu} \backslash\{0\}$ 
verifies the linear relation

$$
b_{1}+a_{1} b_{2}+\left(a_{1} a_{2}\right) b_{3}+\cdots+\left(a_{1} \cdots a_{\nu-1}\right) b_{\nu}=0 .
$$

For each $\tau \in P_{\nu}$ the corresponding (geometric) isomorphism $\varphi_{\tau}: \Gamma \rightarrow \Gamma$ maps each generator $\gamma_{i}$ into a new element of $\Gamma$ conjugated to $\gamma_{i}$. Therefore, we deduce that $\rho\left(\varphi_{\tau}\left(\gamma_{i}\right)\right)$ has the same linear part that $\rho\left(\gamma_{i}\right)$, i.e. $\rho\left(\varphi_{\tau}\left(\gamma_{i}\right)\right)=a_{i} \zeta+b_{i}^{\tau}$ for some constants $b_{i}^{\tau}$ depending on $\tau$ and $a_{1}, \ldots, a_{\nu}, b_{1}, \ldots, b_{\nu}$. We define $\vec{b}^{\tau}=$ $\left(b_{1}^{\tau}, \ldots, b_{\nu}^{\tau}\right) \in \mathbb{C}^{\nu} \backslash\{0\}$.

Proposition 6.1. Using the notations introduced above the following statements hold:

(i) The map $\mathcal{G}(\tau): \mathbb{C}^{\nu} \rightarrow \mathbb{C}^{\nu}$ given by $\mathcal{G}(\tau)(\vec{b})=\vec{b}^{\tau}$ is linear and bijective.

(ii) The map $\mathcal{G}: P_{\nu} \rightarrow \mathrm{GL}(\nu, \mathbb{C})$ is a representation of the pure braid group of the plane.

In order to show this we need to recall some facts concerning $P_{\nu}$. Following [1], we denote the standard generator system of $P_{\nu}$ by $\left\{A_{i j}, 1 \leq i<j \leq \nu\right\}$. The restriction to $P_{\nu}$ of the faithful morphism from the full braid group into $\operatorname{Aut}(\Gamma)$ is given by

$$
\varphi_{A_{i j}}\left(\gamma_{k}\right)= \begin{cases}\gamma_{k} & \text { if } k<i \text { or } k>j \\ \left(\gamma_{i} \gamma_{j}\right) \gamma_{k}\left(\gamma_{i} \gamma_{j}\right)^{-1} & \text { if } k=i \\ \left(\gamma_{i} \gamma_{j} \gamma_{i}^{-1} \gamma_{j}^{-1}\right) \gamma_{k}\left(\gamma_{i} \gamma_{j} \gamma_{i}^{-1} \gamma_{j}^{-1}\right)^{-1} & \text { if } i<k<j \\ \gamma_{i} \gamma_{k} \gamma_{i}^{-1} & \text { if } k=j\end{cases}
$$

Also let us recall the Gassner representation $\mathfrak{g}: P_{\nu} \rightarrow \mathrm{GL}(\nu, \Lambda)$ which maps the generator $A_{i j}$ into the matrix

$$
\left(\begin{array}{ccccccc}
I_{i-1} & 0 & 0 & \cdots & 0 & 0 & 0 \\
0 & 1-x_{i}+x_{i} x_{j} & 0 & \cdots & 0 & x_{i}\left(1-x_{i}\right) & 0 \\
0 & \left(1-x_{i+1}\right)\left(1-x_{j}\right) & 1 & \cdots & 0 & -\left(1-x_{i+1}\right)\left(1-x_{i}\right) & 0 \\
\vdots & \vdots & \vdots & \ddots & \vdots & \vdots & \vdots \\
0 & \left(1-x_{j-1}\right)\left(1-x_{j}\right) & 0 & \cdots & 1 & -\left(1-x_{j-1}\right)\left(1-x_{i}\right) & 0 \\
0 & 1-x_{j} & 0 & \cdots & 0 & x_{i} & 0 \\
0 & 0 & 0 & \cdots & 0 & 0 & I_{\nu-j}
\end{array}\right)
$$

where $\Lambda$ is the $\operatorname{ring} \mathbb{Z}\left[x_{1}^{ \pm 1}, \ldots, x_{\nu}^{ \pm 1}\right]$. It is well-known that Gassner representation is not irreducible. Actually $\vec{v}=\left(1-x_{1}, \ldots, 1-x_{\nu}\right)$ is an eigenvector of $\mathfrak{g}(\tau)$ for any $\tau \in P_{\nu}$. Moreover $W=\left\{\left(\xi_{1}, \ldots, \xi_{\nu}\right) \in \Lambda^{\nu} \mid \xi_{1}+x_{1} \xi_{2}+\cdots+\left(x_{1} \cdots x_{\nu-1}\right) \xi_{\nu}=0\right\}$ is a $\mathfrak{g}$-invariant $\Lambda$-submodule of $\Lambda^{\nu}$ and $\vec{v} \notin W$. The induced representation $\mathfrak{g}^{r}: P_{\nu} \rightarrow \mathrm{GL}(W)$ is irreducible and it is called the reduced Gassner representation.

The condition (3) on the vector $\vec{b}$ can be translated as $\vec{b} \in W_{\rho_{1}}$ where $W_{\rho_{1}} \subset$ $\mathbb{C}^{\nu}$ is the image of $W$ by the specialization morphism $\rho_{1}: \Lambda \rightarrow \mathbb{C}, x_{i} \mapsto a_{i}$ obtained from the linear terms of the affine representation $\rho$. Note that $\rho_{1}$ is injective if and only if $a_{1}, \ldots, a_{\nu}$ are algebraically independent. We will denote $\mathfrak{g}_{\rho_{1}}$ 
and $\mathfrak{g}_{\rho_{1}}^{r}$ the compositions of the Gassner representation and the reduced Gassner representation with the specialization morphism $\rho_{1}$.

Proof of the Proposition 6.1. Let us denote $\vec{c}=\mathcal{G}(\tau)(\vec{b})$ by $\left(c_{1}, \ldots, c_{\nu}\right)$ and also $\vec{e}=\mathcal{G}\left(A_{i j} \tau\right)(\vec{b})$ by $\left(e_{1}, \ldots, e_{\nu}\right)$. Using the definition of $\mathcal{G}$, it is easy to see that $\mathcal{G}\left(A_{i j} \tau\right)(\vec{b})=\mathcal{G}\left(A_{i j}\right)(\mathcal{G}(\tau)(\vec{b}))$, i.e. that $\vec{e}=\mathcal{G}\left(A_{i j}\right)(\vec{c})$. Thus, from (4) follows that

$$
e_{k}=\left\{\begin{array}{cl}
c_{k} & \text { if } k<i \text { or } k>j \\
\left(1-a_{i}+a_{i} a_{j}\right) c_{i}+a_{i}\left(1-a_{i}\right) c_{j} & \text { if } k=i \\
c_{k}+\left(1-a_{k}\right)\left[\left(1-a_{j}\right) c_{i}-\left(1-a_{i}\right) c_{j}\right] & \text { if } i<k<j \\
\left(1-a_{j}\right) c_{i}+a_{i} c_{j} & \text { if } k=j
\end{array}\right.
$$

which we can rewrite as $\mathcal{G}\left(A_{i j} \tau\right)(\vec{b})=\mathfrak{g}_{\rho_{1}}\left(A_{i j}\right)(\mathcal{G}(\tau)(\vec{b}))$. Using that the elements $A_{i j}$ form a system of generators of the group $P_{\nu}$ and applying iteratively the last equality, we deduce that for any $\tau \in P_{\nu}$ we have $\mathcal{G}(\tau)(\vec{b})=\mathfrak{g}_{\rho_{1}}(\tau)(\vec{b})$. Since we know that $\mathfrak{g}_{\rho_{1}}$ is a representation of $P_{\nu}$ into $\mathrm{GL}(\nu, \mathbb{C})$ we conclude (i) and (ii).

Proposition 6.2. Let $\rho$ be an affine non abelian representation of $\Gamma$ given by $\rho\left(\gamma_{i}\right)=a_{i} \zeta+b_{i}$ such that $\vec{b}=\left(b_{1}, \ldots, b_{\nu}\right) \in W_{\rho_{1}} \backslash\{0\}$.

(i) For any $\tau \in P_{\nu}$ we have $\mathcal{G}(\tau)(\vec{b})=\mathfrak{g}_{\rho_{1}}(\tau)(\vec{b})$.

(ii) Assume that $\tau \in M_{\nu}$, then $\tau \in \operatorname{Sim}(\rho)$ if and only if $[\vec{b}] \in \mathbb{P}\left(W_{\rho_{1}}\right) \cong \mathbb{C P}^{\nu-2}$ is a fixed point of the projective transformation induced by $\mathfrak{g}_{\rho_{1}}^{r}(\tau)$.

(iii) The reduced Gassner representation $\mathfrak{g}^{r}$ is faithful on $M_{\nu}$ if and only if any generic affine representation of $\Gamma$ has trivial symmetry group.

Proof. The first assertion is showed in the proof of the Proposition 6.1. For proving (ii) we recall that $\rho\left(\gamma_{\nu+1}\right)$ is linear and $\varphi_{\tau}\left(\gamma_{\nu+1}\right)=\gamma_{\nu+1}$ for every $\tau \in P_{\nu}$. On the other hand, an affine transformation commutes with a linear one only if it is also linear. Thus in the definition of $\operatorname{Sim}(\rho)$ we must take $g=\mu \zeta \in \operatorname{Aff}(1, \mathbb{C})$ and therefore $I_{g}\left(a_{i} \zeta+b_{i}\right)=a_{i} \zeta+\mu b_{i}$. The sufficiency of (iii) is clear because if $\tau \in M_{\nu}$ is in the kernel of $\mathfrak{g}^{r}$ then, by (ii), for any affine representation $\rho$ of $\Gamma$ we should have that $\tau \in \operatorname{Sim}(\rho)$. We now prove that the faithfulness of $\mathfrak{g}^{r}$ on $M_{\nu}$ implies the existence of a residual set of affine representations $\rho$ of $\Gamma$ with trivial symmetry group. Note that if we choose the linear parts $a_{1}, \ldots, a_{\nu} \in \mathbb{C}^{*}$ of $\rho$ algebraically independent (residual set) then $\mathfrak{g}_{\rho_{1}}^{r}$ is also faithful. We denote by $p$ the canonical projection of $\operatorname{GL}(\nu-1, \mathbb{C})$ onto $\operatorname{PGL}(\nu-1, \mathbb{C})$ whose kernel is $\mathbb{C}^{*} I d_{\nu-1}$, i.e. the center of $\operatorname{GL}(\nu-1, \mathbb{C})$. If $\mathfrak{g}_{\rho_{1}}^{r}$ is faithful then $\operatorname{ker}\left(p \circ \mathfrak{g}_{\rho_{1}}^{r}\right) \cong \operatorname{ker} p \cap \mathfrak{g}_{\rho_{1}}^{r}\left(M_{\nu}\right)$ and therefore its elements are contained in the center of $M_{\nu}$, which is trivial. Therefore $p \circ \mathfrak{g}_{\rho_{1}}^{r}$ is also faithful. Thus, for any $\tau \in M_{\nu}, \tau \neq 1$ we have that the fixed point set $F i x(\tau)$ of the projective transformation $\left(p \circ \mathfrak{g}_{\rho_{1}}^{r}\right)(\tau) \in \operatorname{PGL}(\nu-1, \mathbb{C})$ is a proper algebraic variety of $\mathbb{C P}^{\nu-2}$. Since $M_{\nu}$ is countable the set $\mathfrak{R}=\mathbb{C P}^{\nu-2} \backslash$ $\left(\bigcup_{\tau \in M_{\nu} \backslash\{1\}} F i x(\tau)\right)$ is residual in $\mathbb{C P}^{\nu-2}$. If $\left[b_{1}, \ldots, b_{\nu}\right] \in \mathbb{P}\left(W_{\rho_{1}}\right)$ is a point in the 
set $\mathfrak{R}$ then the affine representation $\rho: \Gamma \rightarrow \operatorname{Aff}(1, \mathbb{C})$ given by $\rho\left(\gamma_{i}\right)=a_{i} \zeta+b_{i}$ has trivial symmetry group.

The faithfulness of Gassner representation on $P_{\nu}$ is still an open question which we can reformulate in the following way.

Theorem 6.3. The Gassner representation on $P_{\nu}$ is faithful if and only if any generic affine representation of the free group of rank $\nu$ has trivial symmetry group.

Proof. Gassner representation splits as the trivial representation acting over $\langle\vec{v}\rangle$ plus the reduced Gassner representation acting over $W$. Then $\mathfrak{g}$ is faithful if and only if $\mathfrak{g}^{r}$ is. Furthermore $M_{\nu} \cong P_{\nu} / Z\left(P_{\nu}\right)$ is a non-central normal subgroup of $P_{\nu}$ and Gassner representation on the center $Z\left(P_{\nu}\right)$ of $P_{\nu}$ is injective. Applying the Theorem 2.2 of [7] we deduce that $\mathfrak{g}$ is faithful if and only if $\mathfrak{g}_{\mid M_{\nu}}^{r}$ is.

However, it is well-known that for $\nu=3$ Gassner representation is faithful, see for instance [1].

Corollary 6.4. The symmetry group of a generic affine representation of the free group of rank 3 is trivial.

Theorem 6.5. Let $\mathcal{F}_{0}$ be a N.A.G. foliation of order $\nu=3$ with holonomy representation $H_{0}$ and residues $\alpha_{1}, \ldots, \alpha_{4}$ such that three of the numbers $e^{2 i \pi \alpha_{1}}, \ldots$, $e^{2 i \pi \alpha_{4}}$ are algebraically independent.

(i) If $\mathcal{F}_{0}$ is generic then $\operatorname{Sim}\left(H_{0}\right)$ is trivial.

(ii) If $\operatorname{Sim}\left(H_{0}\right)$ contains a non trivial element then $\operatorname{Sim}\left(H_{0}\right) \cong \mathbb{Z}$. Moreover there are examples of foliations $\mathcal{F}_{0}$ with a prescribed non trivial symmetry for $H_{0}$.

Proof. The first assertion is consequence of Corollary 6.4. Let $\rho$ be the affine representation associated to $H_{0}$. The assumption on the linear part of $H_{0}$ implies that $\rho_{1}: \Lambda \rightarrow \mathbb{C}$ is injective. Therefore $p \circ \mathfrak{g}_{\rho_{1}}^{r}$ is a isomorphism of $M_{3}$ onto a subgroup of PGL $(2, \mathbb{C})$. Since $M_{3}$ is isomorphic to the free group of rank two we have that $\operatorname{Sim}(\rho)$ is also a free group. On the other hand, the image of $\operatorname{Sim}(\rho)$ by $p$ o $\mathfrak{g}_{\rho_{1}}^{r}$ is contained in the isotropy subgroup of a point $[\vec{b}] \in \mathbb{C P}^{1}$, which is isomorphic to the affine group $\operatorname{Aff}(1, \mathbb{C})$. Therefore $\operatorname{Sim}(\rho)$ is solvable and we conclude that it is either trivial or infinite cyclic. Finally, $\operatorname{Sim}\left(H_{0}\right)$ is a subgroup of $\operatorname{Sim}(\rho)$ therefore it verifies the same dichotomy. The last assertion is a consequence of Lins Neto's theorem stated in section 5.1. Indeed, given $\tau \in M_{\nu}$ we can fix an affine representation with $[\vec{b}] \in F i x(\tau)$, and then to realize this affine representation as the holonomy representation of a holomorphic homogeneous foliation in $\left(\mathbb{C}^{2}, 0\right)$. 


\section{The quasi-homogeneous case}

We have seen how to identify the moduli space of singular germs of homogeneous foliations whose first non-zero jet is generic enough. But, what about germs with degenerate first non-zero jet? An illustrative example of this situation is given by a singular germ of foliation whose linear part is nilpotent, i.e. both eigenvalues vanish. This case has been treated, among others, by R. Moussu in [12] and F. Loray in [8]. Consider a 1-form $\omega=\omega_{1}+\omega_{2}+\cdots$ with nilpotent linear part $\omega_{1}=$ $y d y$ and generic quadratic part $\omega_{2}$. It is known that $\omega$ is analytically conjugated to $\omega_{\Delta}=d\left(y^{2}-x^{3}\right)+\Delta(x, y)(2 x d y-3 y d x)$ with $\Delta(0,0)=0$. From this we see that the cusp $y^{2}-x^{3}=0$ is a separatrix of $\omega_{\Delta}$ and this is the reason to call it a cuspidal singularity. See for instance [8], where the following result is proved.

Theorem (Loray). Cuspidal singularities are topologically rigid, i.e. if $\omega \sim_{\text {top }} \omega_{\Delta}$ then $\omega \sim_{\text {an }} \omega_{\Delta}$.

Both homogeneous and cuspidal singularities are particular cases of a more general situation: quasi-homogeneous singularities.

Definition 7.1. Let $\mathcal{F}$ be a holomorphic germ of a non-dicritical foliation and let $f$ be a reduced equation of the set of its sepatrices. We say that $\mathcal{F}$ is quasihomogeneous if $f$ itself is a quasi-homogeneous function, i.e. $f(x, y)$ belongs to its jacobian ideal $\left(\frac{\partial f}{\partial x}, \frac{\partial f}{\partial y}\right)$.

Remark 7.2. This definition is independent of the choice of the coordinates but it is equivalent to the existence of a privileged coordinate system $(x, y)$ such that

$$
f(x, y)=\sum_{\alpha i+\beta j=d} a_{i j} x^{i} y^{j}, \quad \text { for some positive integers } \alpha, \beta \text { and } d .
$$

Moreover, if $\mathcal{F}$ is a generalized curve given by a 1 -form $\omega$ then we can write it as $\omega=d f+H(x, y)(\alpha x d y-\beta y d x)$, see [10]. The integers $\alpha$ and $\beta$ can be taken coprime and they are called the weights of $\mathcal{F}, \omega$ or $f$. For instance, homogeneous and cuspidal singularities are quasi-homogeneous foliations whose weights are $(1,1)$ and $(2,3)$ respectively.

It follows from (6) that $\operatorname{Sep}(\mathcal{F})$ is the union of $d$ leaves of the dicritical foliation $\mathcal{R}_{\alpha \beta}$ given by the 1 -form $\alpha x d y-\beta y d x$, which admits $\frac{y^{\alpha}}{x^{\beta}}$ as a meromorphic first integral. In the sequel we will assume that $\mathcal{F}$ is a generalized curve. Therefore, the reduction of singularities of $\mathcal{F}$ coincides with the desingularization of $\operatorname{Sep}(\mathcal{F})$, or even of $\mathcal{R}_{\alpha \beta}$. We exclude the cases $\alpha=1$ and $\beta=1$ in which the separatrices of $\mathcal{F}$ are smooth. Let $U$ be an open set containing the origin where the germ $\mathcal{F}$ is well defined and denote by $\pi:(\widetilde{U}, E) \rightarrow(U, 0)$ the composition of blowing-ups reducing the singularities of $\mathcal{F}$ and $\widetilde{\mathcal{F}}$. There is a distinguished irreducible component $D$ of 
$E$ which carries on the singular separatrices of $\mathcal{F}$. In the Figure 1 is described the exceptional divisor in the case $\alpha=2$ and $\beta=3$ and the separatrices of $\widetilde{\mathcal{F}}=\pi^{*} \mathcal{F}$ when $d=3$. The others cases have a similar exceptional divisor adding new irreducible components between $D$ and those that contain the axes. These are added according to the euclidian algorithm to find the greater common divisor of $\alpha$ and $\beta$. The lines $\{x=0\}$ or $\{y=0\}$ could be separatrices of $\mathcal{F}$ but we will also exclude this case. It turns out that the coins of $E$ are singularities of $\widetilde{\mathcal{F}}$ which admit local holomorphic first integrals. Therefore, their holonomy transformations are periodic.

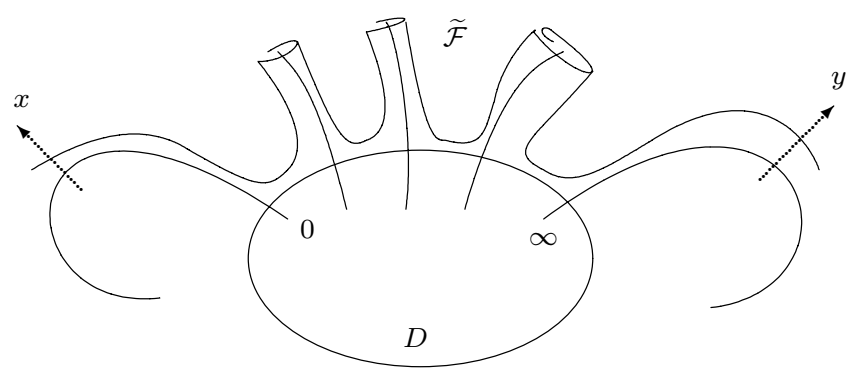

FIG. 1. The reduction of singularities of a quasi-homogeneous foliation with weights $\alpha=2$, $\beta=3$ and $d=3$ separatrices.

Let $b$ be a point in $D^{*}=D \backslash \operatorname{Sing}(\widetilde{\mathcal{F}})$ and denote by $\Sigma$ the fiber of $\mathcal{R}_{\alpha \beta}$ over $b$. We consider the holonomy representation $\operatorname{Hol}_{\mathcal{F}}^{D}: \pi_{1}\left(D^{*}, b\right) \rightarrow \operatorname{Diff}(\Sigma, b)$ of the leaf $D^{*}$ of $\widetilde{\mathcal{F}}$. Let $\gamma_{0}, \gamma_{1}, \ldots, \gamma_{d}, \gamma_{\infty}$ be a geometric generator system of $\pi_{1}\left(D^{*}, b\right)$ with $\gamma_{0} \gamma_{1} \cdots \gamma_{d} \gamma_{\infty}=1$. If follows from the above discussion that $h_{0}=\operatorname{Hol}_{\mathcal{F}}^{D}\left(\gamma_{0}\right)$ and $h_{\infty}=\operatorname{Hol}_{\mathcal{F}}^{D}\left(\gamma_{\infty}\right)$ are periodic. More precisely, $h_{0}^{\alpha}=h_{\infty}^{\beta}=i d$. We define $N_{\alpha \beta}$ as the normal subgroup of $\pi_{1}\left(D^{*}, b\right)$ generated by $\gamma_{0}^{\alpha}$ and $\gamma_{\infty}^{\beta}$, which does not depend on the choice of the generators $\gamma_{0}, \ldots, \gamma_{\infty}$. We also denote by $\Gamma$ the quotient of $\pi_{1}\left(D^{*}, b\right)$ by $N_{\alpha \beta}$ and $H_{\mathcal{F}}: \Gamma \rightarrow \operatorname{Diff}(\Sigma, b)$ the morphism induced by $\operatorname{Hol}_{\mathcal{F}}^{D}$.

Definition 7.3. A quasi-homogeneous N.A.G. foliation is a generalized curve $\mathcal{F}$ whose separatrices are a finite number of cuspidal fibres of $\mathcal{R}_{\alpha \beta}$ such that

(i) the singularities of $\widetilde{\mathcal{F}}$ on $D \backslash\{0, \infty\}$ are hyperbolic and their residues are all different, generating a dense additive subgroup of $\mathbb{C}$;

(ii) the holonomy group of $\mathcal{F}$ with respect to $D$ is non abelian.

Remark 7.4. N.A.G. conditions for quasi-homogeneous foliations imply that $\alpha, \beta$ are greater than one and that the number of separatrices is $d>2$. As well as in the 
homogeneous case, the conditions (i) and (ii) also imply the topological rigidity of the holonomy group of $\mathcal{F}$ respect to $D$.

Definition 7.5. Let $\mathcal{F}_{0}$ be a quasi-homogeneous germ of foliation. The moduli space of $\mathcal{F}_{0}$ relative to the quasi-homogeneous class is defined by

$$
\mathcal{M}_{\mathcal{Q H}}\left(\mathcal{F}_{0}\right)=\left\{\mathcal{F} \text { quasi-homogeneous germ of foliation } \mid \mathcal{F} \sim_{\text {top }} \mathcal{F}_{0}\right\} / \sim_{\text {an }} .
$$

Theorem F. Let $\mathcal{F}_{0}$ be a N.A.G. quasi-homogeneous germ of foliation with $d>2$ separatrices. Then $\mathcal{M}_{\mathcal{Q H}}\left(\mathcal{F}_{0}\right)$ is a connected covering of $F_{3, d-1}\left(\mathbb{S}^{2}\right)$ whose fundamental group is completely determined by the holonomy representation $H_{\mathcal{F}_{0}}$.

The proof follows from the analytic and topological classifications below using a similar construction as in section 5 . We can find the analytic classification in [12] and [3]. Although the original proof was given for $\alpha=2, \beta=3$ and $d=1$ the techniques apply to the general case:

Theorem (Moussu). Let $\mathcal{F}$ and $\mathcal{F}^{\prime}$ be two germs of N.A.G. quasi-homogeneous foliations with the same weights and the same number of separatrices. Then $\mathcal{F}$ is analytically conjugated to $\mathcal{F}^{\prime}$ if and only if the following conditions hold:

(i) there exists a biholomorphic transformation $\phi: D \rightarrow D$ fixing 0 and $\infty$ such that $\phi(\operatorname{Sing}(\widetilde{\mathcal{F}}) \cap D)=\operatorname{Sing}\left(\widetilde{\mathcal{F}^{\prime}}\right) \cap D$ and the residues of $\widetilde{\mathcal{F}}$ and $\widetilde{\mathcal{F}^{\prime}}$ at the singularities corresponding by $\phi$ are equal;

(ii) there is a germ of biholomorphism $\psi:(\Sigma, b) \rightarrow\left(\Sigma^{\prime}, b^{\prime}\right)$ verifying $\psi_{*} \circ H_{\mathcal{F}}=$ $H_{\mathcal{F}^{\prime}} \circ \varphi$, where $\varphi: \Gamma \rightarrow \Gamma^{\prime}$ is the isomorphism induced by $\phi$.

Finally, we devote the rest of the section to the topological classification of quasi-homogeneous N.A.G. foliations.

Lemma 7.6. Let $S \subset U$ be $d>0$ different cuspidal fibres of the fibration $\mathcal{R}_{\alpha \beta}$ restricted to the open set $U$. Then the fundamental group of $U^{*}=U \backslash S$ can be presented as follows:

$$
\left\langle g_{0}, g_{1}, \ldots, g_{d}, g_{\infty} \mid g_{0} g_{1} \cdots g_{d} g_{\infty}=1, g_{0}^{\alpha}=g_{\infty}^{\beta}=c,\left[c, g_{j}\right]=1, j=1, \ldots, d\right\rangle .
$$

Moreover, its centre is infinite cyclic generated by $c$ and these generators can be chosen in such a way that the mapping $\gamma_{j} \mapsto g_{j}$ induces a isomorphism of $\Gamma$ onto $\pi_{1}\left(U^{*}\right) /\langle c\rangle$.

The proof consists in an iterated application of Seifert-Van Kampen Theorem. Now, we can formulate the topological classification theorem for quasihomogeneous N.A.G. foliations.

Theorem 7.7. If $\Phi:\left(\mathbb{C}^{2}, 0\right) \rightarrow\left(\mathbb{C}^{2}, 0\right)$ is a germ of homeomorphism conjugating two quasi-homogeneous N.A.G. singularities $\mathcal{F}$ and $\mathcal{F}^{\prime}$ then $\Phi$ induces a geometric 
isomorphism $\varphi: \Gamma \rightarrow \Gamma^{\prime}$ and a germ of biholomorphism $\psi:(\Sigma, b) \rightarrow\left(\Sigma^{\prime}, b^{\prime}\right)$ making commutative the following diagram:

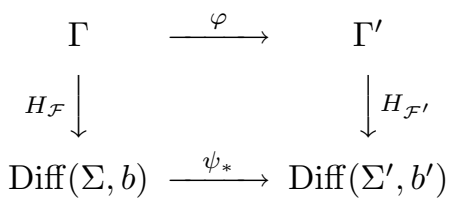

Furthermore, the residues of $\mathcal{F}$ and $\mathcal{F}^{\prime}$ along the separatrices corresponding by $\Phi$ are equal. Conversely, if there are a geometric isomorphism $\varphi$ and a germ of biholomorphism $\psi$ as before then there is a germ of homeomorphism $\Phi:\left(\mathbb{C}^{2}, 0\right) \rightarrow$ $\left(\mathbb{C}^{2}, 0\right)$ which preserves the fibres of $\mathcal{R}_{\alpha \beta}$ and conjugates $\mathcal{F}$ to $\mathcal{F}^{\prime}$.

Sketch of the proof. The isomorphism $\Phi_{*}: \pi_{1}\left(U^{*}\right) \rightarrow \pi_{1}\left(U^{* *}\right)$ maps center onto center. Therefore it induces an isomorphism $\varphi: \Gamma \rightarrow \Gamma^{\prime}$ on the quotient. We construct a germ of homeomorphism $\psi:(\Sigma, b) \rightarrow\left(\Sigma^{\prime}, b^{\prime}\right)$ conjugating the holonomy groups of $\mathcal{F}$ and $\mathcal{F}^{\prime}$ as in section 3. Since the holonomy group of $\mathcal{F}$ is rigid we conclude that $\psi$ is actually holomorphic. For the converse, $\Phi$ can be built using the lifting path method as in Theorem E.

\section{References}

[1] Joan S. Birman, Braids, links, and mapping class groups, Annals of Mathematics Studies, No. 82, Princeton University Press, Princeton, N.J., 1974.

[2] César Camacho, Alcides Lins Neto and Paulo Sad, Topological invariants and equidesingularization for holomorphic vector fields, J. Differential Geom. 20 (1) (1984), 143-174.

[3] D. Cerveau and R. Moussu, Groupes d'automorphismes de $(\mathbf{C}, 0)$ et équations différentielles $y d y+\cdots=0$, Bull. Soc. Math. France 116 (4) (1989), 459-488.

[4] Dominique Cerveau and Paulo Sad, Problèmes de modules pour les formes différentielles singulières dans le plan complexe, Comment. Math. Helv. 61 (2) (1986), 222-253.

[5] Yu. S. Ilyashenko, Topology of phase portraits of analytic differential equations on a complex projective plane, Trudy Sem. Petrovsk. 4 (1978), 83-136.

[6] Alcides Lins Neto, Construction of singular holomorphic vector fields and foliations in dimension two, J. Differential Geom. 26 (1) (1987), 1-31.

[7] D. D. Long. A note on the normal subgroups of mapping class groups. Math. Proc. Camb. Phil. Soc., 99:79-87, 1986.

[8] Frank Loray, Rigidité topologique pour des singularités de feuilletages holomorphes, in: Ecuaciones Diferenciales. Singularidades, 213-234, Universidad de Valladolid, 1995.

[9] J.-F. Mattei and R. Moussu, Holonomie et intégrales premières, Ann. Sci. École Norm. Sup. (4) 13 (4) (1980), 469-523.

[10] Jean-François Mattei, Quasi-homogénéité et équiréductibilité de feuilletages holomorphes en dimension deux, Astérisque (261) xix (2000), 253-276. Géométrie complexe et systèmes dynamiques (Orsay, 1995).

[11] Jean-François Mattei and Eliane Salem, Complete systems of topological and analytical invariants for a generic foliation of $\left(\mathbf{C}^{2}, 0\right)$, Math. Res. Lett. 4 (1) (1997), 131-141.

[12] Robert Moussu, Holonomie évenescente des équations différentielles dégénerées transverses, in: Singularities and dynamical systems (Iráklion, 1983), 161-173, North-Holland, Amsterdam, 1985. 
[13] Heiner Zieschang, Elmar Vogt and Hans-Dieter Coldewey, Surfaces and planar discontinuous groups, Springer, Berlin, 1980. Translated from the German by John Stillwell.

\section{David Marín}

Departament de Matemàtiques

Universitat Autònoma de Barcelona

E-08193 Bellaterra

Barcelona

Spain

e-mail: david@mat.uab.es

(Received: December 14, 2001)

$$
\begin{aligned}
& \text { To access this journal online: } \\
& \text { (4P) http://www.birkhauser.ch }
\end{aligned}
$$

